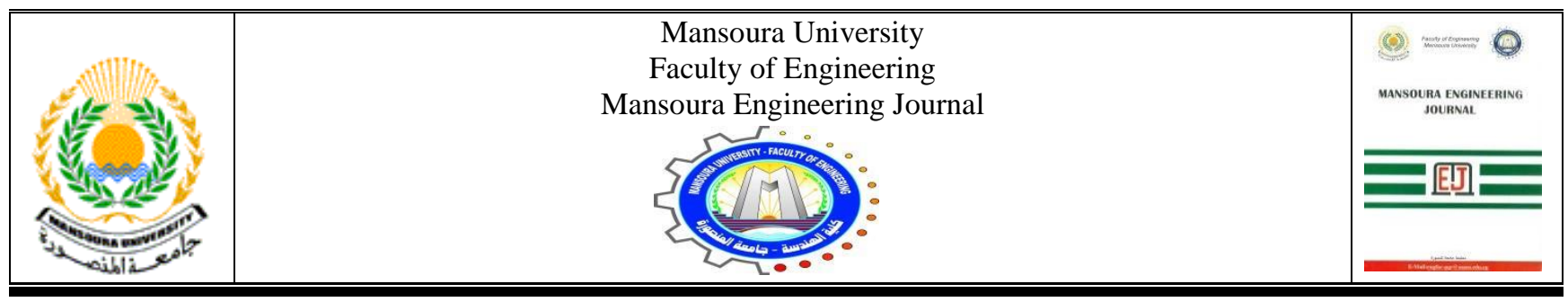

\title{
Analysis of inductive characteristics for various helical and spiral coil configurations supported by COMSOL Multiphysics
}

\author{
Ahmed M. Elbeshbeshy, Mohammad E. M. Rizk and Sahar Sidky Kaddah
}

\author{
KEYWORDS: \\ wireless power transfer, \\ misalignment, \\ electromagnetics, helical \\ coil, spiral coil, charging \\ pads.
}

\begin{abstract}
Wireless power transfer system has a major concern for several decades since various benefits have been included against wired counterpart. In this context, coupling structure has been investigated for various coils having different shapes of both magnetic cores and windings. This investigation is implemented based on a mathematical and modeling analysis to calculate inductive values of charging setups. This paper provides an accurate evaluation of inductive characteristics for the proposed coreless helical and spiral coils. The evaluation considers the dependence of inductive parameters on distance, geometry, and misalignment of coupling coils. In addition, the evaluation method is verified by the COMSOL software. Moreover, an accurate approximation of required inductive parameters is provided for the design of wireless charging system. Furthermore, four ferrite cores are provided for both winding types; and their coupling coefficients are examined to show the best configuration. Results show the validity of both theoretical and simulated analysis.
\end{abstract}

\section{INTRODUCTION}

$\mathrm{T}$ Transports industry is a growing user of fossil fuels with internal combustion engines (ICE). Nowadays, electric vehicles (EVs) are increasingly becoming the main replacement for ICE vehicles [1]. The significant reduction in local emissions and greenhouse gases are key benefits of EVs [2], [3]. Wireless power transfer system (WPTS) with essential power transmission features is developed for charging EVs [4], [5]. Spacing between any charging coils is a key problem in the design of a WPTS. Coil

Received: (28 August, 2020) - Revised: (30 December, 2020) - Accepted: (12 March, 2021)

*Corresponding author: Ahmed M. Elbeshbeshy, Electrical Engineering Department, Mansoura University, El-Mansoura, 36615, Egypt (e-mail: ahmedbesh26@mans.edu.eg).

Mohammad E. M. Rizk, Electrical Engineering Department, Mansoura University, El-Mansoura, 36615, Egypt, (e-mail: eng_mohammad2007@mans.edu.eg).

Prof. Sahar S. Kaddah, Electrical Engineering Department, Mansoura University, El-Mansoura, 36615, Egypt, (e-mail: : skaddah@mans.edu.eg). arrangement consists of the transmitting and receiving unit, the first is stationary below road and is supplied from grid, and the latter is placed at vehicle base and provides energy to onboard rechargeable battery [6]. Compensation network is used as a method for overcoming high losses in transmitter and receiver coils, which includes series or parallel capacitors used to transfer only active power instead of using a large primary current to transfer the same amount [7], [8].

Another approach for increasing performance involves improvement of coil coupling parameters such as transmitter and receiver coil diameter, core material, size, shape, and position of coil winding and magnetic core [9], [10]. In this context, an accurate calculation of inductive characteristics is essential for the coil design to transfer required capacity of power. Various literatures have analyzed the calculation of self and mutual inductance between two circular coreless coils [11]-[19]. Many of the contributions have been based on application of Maxwell's formula [11], Neumann formula, and Biot-Savart law [12].

Self-inductance can be directly evaluated by solving Maxwell's equations. A three-dimensional (3D) finite element 
software, such as COMOSOL [20], can be used to provide another reliable numerical solution. However, 3D software programs are computationally complex and time-consuming, making them more suitable for prototype validation than for inductor design. The greenhouse strategy is another way used to evaluate inductance [13]. This technique provides adequate precision and speed, but inductor design can be provided indirectly. In order to design and optimize inductive parameters for charging setups, three approaches are highly desirable. In the first approach, some approximate expressions can be used such as the formula given by Wheeler [14]. In the second approach, electromagnetic theories are used to develop an approximate expression which describes the sides of spiral coils as current sheets. Furthermore, self and mutual inductances are evaluated by using geometric mean distance, arithmetic mean distance, and arithmetic mean square distance principles [11]. For the third approach, a monomial approximation is obtained from the integration of a wide database of inductors. The mentioned approximations are accurate, with normal 2-3\% errors, and simple [15] .

Mutual inductance of circular coils can be evaluated in analytical or semi-analytical "magnetic vector potential mathematical approach" methods described over elliptic integrals of the first, second, and third kind [16], [17], Heuman's Lambda function, Legendre functions, and Bessel functions [18]. Grover developed a formula for the evaluation of mutual inductance between circular filaments placed at any distance with respect to the other [19]. Furthermore, it is possible to evaluate the mutual inductance between circular coils using numerical methods, such as the finite element method and the boundary element method. These numerical operations are time consuming and computationally complex [12]. A simplified analytical model of mutual inductance evaluation is essential for the design and optimization of WPTS, which does not require any numerical techniques. The presented method predicts geometrical parameters and relative positioning of the coils. Hence, the computing model of mutual inductance will facilitate analytical estimation and optimization of the design characteristics for WPTS.

The main contribution of this paper is to investigate inductive characteristics, including 1) self-inductance; 2) mutual inductance; and 3) coupling coefficient, depending on 1) coil distance; 2) axial and angular misalignment; 3) turn number and 4) turn distance for various coil configurations. Coil misalignment, as well as coil distance, can vary from the designed values and these variations significantly change WCS coupling coefficient. Furthermore, the transfer power capacity and efficiency are reduced [21]. Formulas of inductive characteristics for coreless coupling structure are evaluated to obtain valuable guidance on their value and are utilized to validate the simulated results by COMSOL software. Then an accurate analysis is carried out for identical transmitter and receiver coil arrangement with the help of COMSOL software for a helical and spiral coil with core and coreless [20].

This paper is divided into five sections. Section II introduces formulas of the inductive characteristics for a noncore coil coupling system. Section III uses COMSOL software to investigate dependence of inductive characteristics on coil parameters for the previous section. The use of ferrite cores is included in section IV to enhance the analysis of the preceding sections. Moreover, Section V concludes the outcomes of this paper.

\section{BASIC ANALYSIS OF HELICAL AND SPIRAL COILS}

Coil and magnetic core shapes are critical in improving WPTS performance and in solving problems of horizontal and angular misalignment. Coupling coils are commonly classified into two patterns: polarized and non-polarized pads. Nonpolarized pads consist of single-coil shapes, including circular pads (CPs) as well as rectangular pads, that only produce the vertical flux component. On the other hand, polarized pads consist of various coil shapes such as H-shaped pads, doubleD pads, double-D quadrature pads, and bipolar pads as seen in Fig. 1, which contain both horizontal and vertical flux components [2], [22]. CPs are the most popular and widely utilized to charge EV for static WPT since the eddy current in this arrangement is held at a minimum value, where sharp edges do not exist. Besides, CPs can exist in a shape of helical or spiral winding in which misalignment tolerance in any direction is the same instead of other types of pads [23].

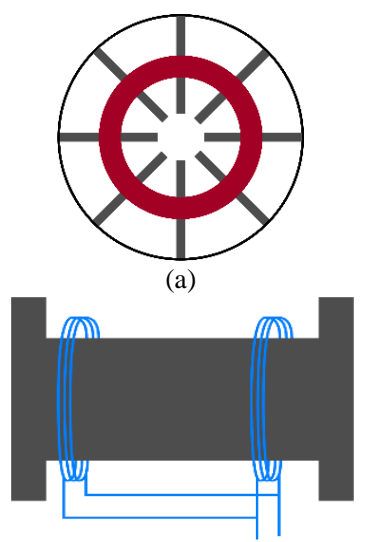

(c)

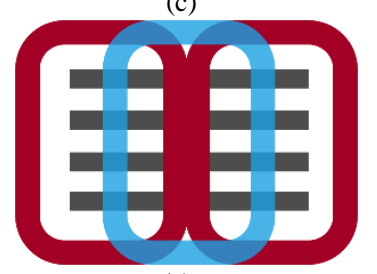

(e)

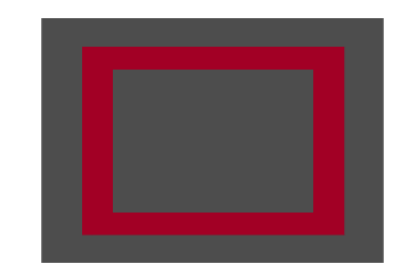

(b)

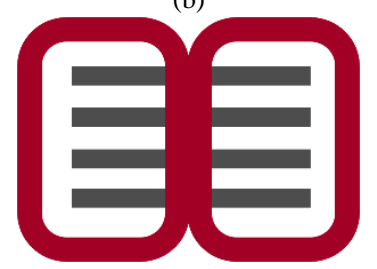

(d)

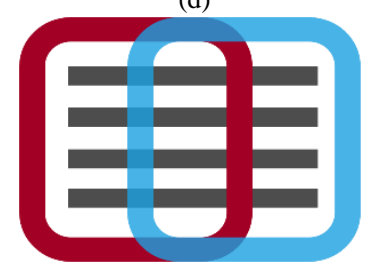

(f)
Fig. 1. Various charging pads like (a) Circular Pad, (b) Rectangular Pad, (c) H-shaped Pad, (d) double-D Pad, (e) double-D quadrature Pad , and (f) bipolar Pad.

Ferromagnetic cores have a variety of features on both transmitter and receiver parts for static WCS, which improve the distribution of magnetic flux and minimize leakage fluxes. Moreover, coupling between coils increases so that fewer turns are required in order to achieve the same self-inductance 
of non-core structure [24]. Design of magnetic core is based on several parameters, such as shape, size, permeability, cost, and operating frequency. Commonly known ferrite shapes such as circular and square I-core, U-core, and E-core are used in the WCS for EV as shown in Fig. 2 [8], [23].

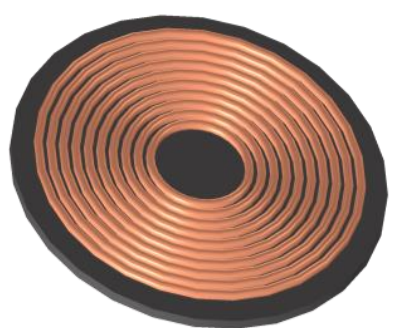

(a)

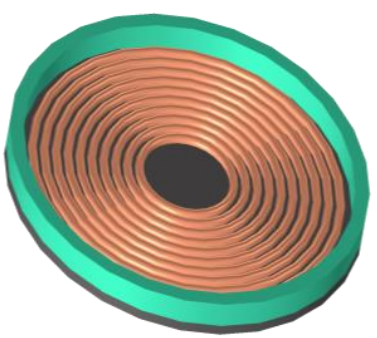

(c)

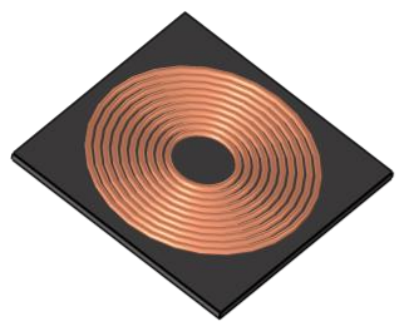

(b)

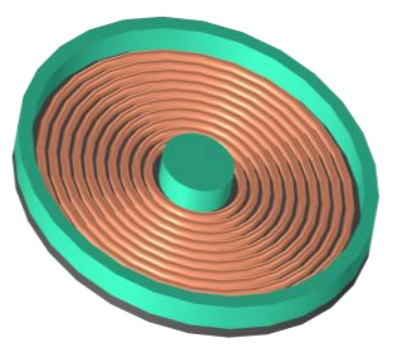

(d)
Fig. 2. Four ferrite core such as (a) Circular I-core, (b) Square I-core, (c) U-core, and (d) E-core.

Magnetic characteristics are derived from the basic electromagnetic theory for the following assumptions: 1) both transmitter and receiver coils, whether helical or spiral as shown in Fig. 3 and Fig. 4, have the same parameters as the number of turns, $\mathrm{N}$, coil material, dimensions, and geometry, 2) neglect coil wire radius compared to coil diameter, 3) Constant magnetic flux density at each point of the sectional coil area, and 4) magnetic field lines assumed to be perpendicular to the coupling arrangement.

\section{A. Magnetic Flux Density}

The amount of magnetic flux through a unit area taken perpendicular to magnetic flux direction is called magnetic flux density. Also, it can be defined as a vector quantity associated with the magnetic field via magnetic permeability that describes the medium in which the conductor current, $I$, is located. In addition, its magnitude at any point among both the transmitting and receiving air coils of radius $R$ and separated by distance $d$ from its cente $\mathrm{r}$ is given by (1), according to the second assumption [25].

$$
B=\frac{\mu_{o}}{4 \pi} N I \frac{2 \pi R^{2}}{\left(R^{2}+d^{2}\right)^{\frac{3}{2}}}
$$

\section{B. Self-Inductance of Helical and Spiral Coil}

Two helical coil turns are bundled as a rectangular area with a wire diameter of $r w$, width of $h_{p}$ and $h_{s}$, length $a$ and $b, N_{p}, N_{s}$ are the turns number of primary and secondary coils, respectively. Besides as shown in Fig. 3 (a), $R_{1}$ and $R_{3}$ represent the radius of the inner coil, as well as $R_{2}$ and $R_{4}$ represent the outer radius. Consequently, each coil selfinductance can be given as in (2) [14].

$$
L_{\text {Helical }}=\frac{31.49\left(R_{p} \times N_{p}\right)^{2}}{6\left(R_{p}\right)+9 a+10 h p}
$$

The average coil radius $R_{p}, R_{s}$ are defined as $R_{p}=$ $0.5\left(R_{1}+R_{2}\right)$ and $R_{S}=0.5\left(R_{3}+R_{4}\right)$ for the primary and secondary coils.

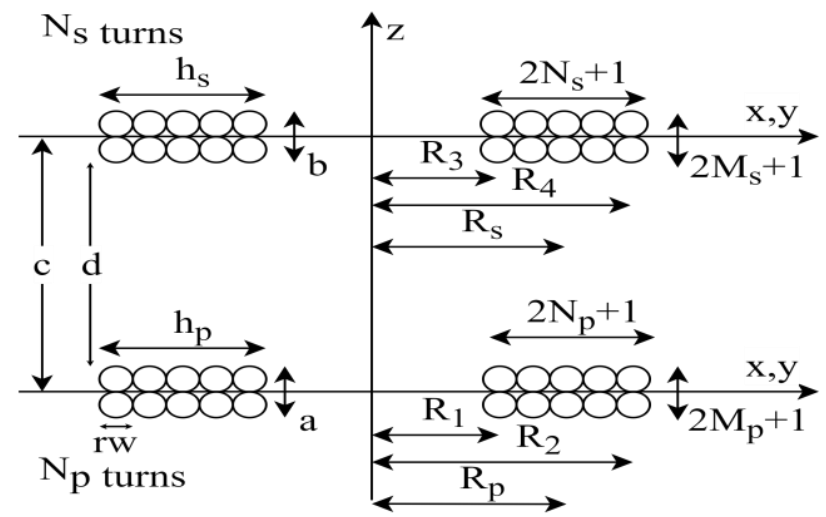

(a)

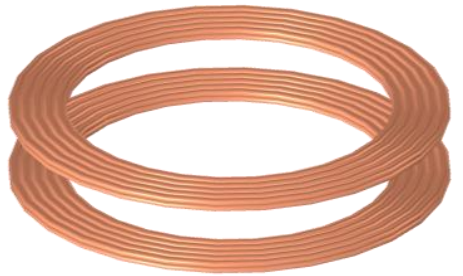

(b)

Fig. 3. (a) Helical coil-coupling layout. (b) Simulated structure of helical coil-coupling.

The difference between the spiral and helical coils is that the spiral coils have only one layer of winding. Moreover, their turns are separated from each other by a sufficient distance. Self-inductance for two-coupling spiral coils can be written in the following form [15]:

$$
L_{\text {Spiral }}=\mu_{o} N_{p}{ }^{2} C_{1} R_{p}\left[\ln \left(C_{2} / \rho\right)+C_{3} \rho+C_{4} \rho^{2}\right]
$$

Where the coefficient values are $C_{1}=1.00, C_{2}=$ 2.46, $C_{3}=0.00, C_{4}=0.20$ depending on the circular layout as seen in Fig. 4 (a). Furthermore, the fill ratio $\rho$ is defined as $\rho=\left(R_{2}-R_{1}+r w\right) /\left(R_{1}+R_{2}\right)$.

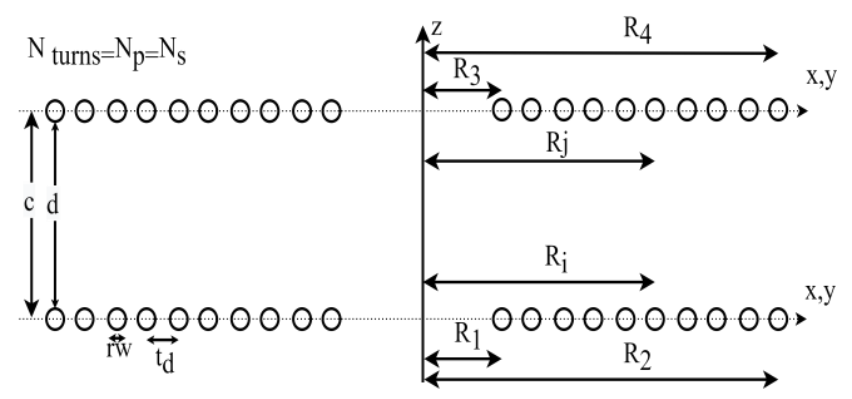

(a) 


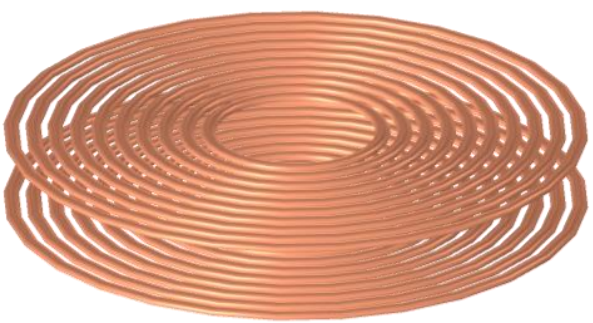

(b)

Fig. 4. (a) Spiral coil-coupling layout. (b) Simulated structure of spiral coil-coupling.

\section{Mutual inductance of Helical and Spiral Coil}

Electromotive force (EMF) is induced in coil by a change of current in another nearby coil. This phenomenon is called electromagnetic induction that depends on mutual inductance, which is a crucial factor in the WPTS design. According to assumptions 3),4) and using the filament method, formula (4) is given by [12], [26] and describes the mutual inductance of the system shown in Fig. 3 (b). The system of two coaxial helical coils is at distance $c$ between the plans of the coils, as shown in Fig. 3 (a). The cross-sectional area of the primary is segmented into $\left(2 M_{p}+1\right)$ by $\left(2 N_{p}+1\right)$ cells and the secondary into $\left(2 M_{s}+1\right)$ by $\left(2 N_{s}+1\right)$ cells.

$$
\begin{array}{r}
M_{\text {Helical }}=\frac{N_{p} N_{s}}{\left(2 M_{p}+1\right)\left(2 N_{p}+1\right)\left(2 M_{s}+1\right)\left(2 N_{s}+1\right)} \\
\times \sum_{g=-M_{p}} \sum_{h=-N_{p}}^{M_{p}} \sum_{q=-M_{s}}^{N_{p}} \sum_{l=-N_{s}}^{N_{s}} M_{i j(g, h, q, l)}
\end{array}
$$

Where,

$$
\begin{gathered}
M_{i j(g, h, q, l)}=\mu_{0} \sqrt{R_{p}(h) R_{s}(l)}\left[\left(\frac{2}{k}-k\right) K(k)-\frac{2}{k} E(k)\right] \\
k=2 \sqrt{\frac{R_{p}(h) R_{s}(l)}{\left(R_{p}(h)+R_{s}(l)\right)^{2}+(d(g, q))^{2}}} \\
R_{p}(h)=R_{p}+\frac{h_{p}}{2 N+1} h, \quad R_{s}(l)=R_{S}+\frac{h_{s}}{2 n+1} l \\
d(g, q)=c-\frac{a}{2 K+1} g+\frac{b}{2 m+1} q \\
h_{s}=R_{4}-R_{3}+r w, \quad h_{p}=R_{2}-R_{1}+r w
\end{gathered}
$$

Furthermore, $K(k)$ and $E(k)$ are the first and second forms of the complete elliptic integrals, respectively [20]. Where $K(k)=\int_{0}^{\pi / 2}\left(1-k^{2} \sin ^{2} \theta\right)^{-1 / 2} d \theta \quad$ and $\quad E(k)=$ $\int_{0}^{\pi / 2}\left(1-k^{2} \sin ^{2} \theta\right)^{1 / 2} d \theta$.

The mutual inductance of the spiral coils shown in Fig. 4 (b) is evaluated using the approximated expression in [27]. The error that existed for this approximated expression (5) is merely $0.038 \%$, when the distance between coils plane is smaller than the inner radius of each coil, compared to the exact solution of Neumann's equation between two current-carrying filaments [19].

$$
\begin{gathered}
M_{\text {Spiral }}=\sum_{i=1}^{N_{p}} \sum_{j=1}^{N_{s}} M_{i j}, \\
M_{i j}=\frac{\mu_{0} \pi R_{i}{ }^{2} R_{j}{ }^{2}}{2\left({R_{i}}^{2}+R_{j}{ }^{2}+c^{2}\right)^{3 / 2}}\left(1+\frac{15}{32} \gamma_{i j}{ }^{2}-\frac{315}{1024} \gamma_{i j}{ }^{4}\right)
\end{gathered}
$$

Where $\quad R_{i}=R_{2}-t_{d}(i-1), \quad R_{j}=R_{4}-t_{d}(j-1)$, $\gamma_{i j}=2 R_{i} R_{j} /\left(R_{i}{ }^{2}+R_{j}{ }^{2}+c^{2}\right)$ and $t_{d}$ is the distance between coil turns.

\section{Coupling Coefficient}

Assessment of the inductive coupling performance is based on evaluating the coupling coefficient. The coupling coefficient $K$ between two coils is given by,

$$
K=\frac{M}{\sqrt{L_{1} L_{2}}}
$$

Equations (1) - (6) provide the estimated values for inductive parameters of non-core helical and spiral coils. Furthermore, it is important to provide proper hints about the reliance of inductive characteristics on layout and geometry of various coils.

\section{INDUCTIVE CHARACTERISTICS OF NON- CORED COILS}

COMSOL software is used in this section to provide a precise estimation of the inductive parameters which strongly support the theoretical analysis in previous section. Furthermore, a small model for the helical and spiral coil arrangement is designed to develop a WPTS based on both geometrical parameters and various coil alignment listed in Table I.

TABLE I

WPTS HELICAL AND SPIRAL COIL SPECIFICATIONS

\begin{tabular}{c||c||c}
\multicolumn{1}{c||}{ Parameter } & Symbol & Value \\
\hline Wire diameter & $r w$ & $1.15 \mathrm{~mm}$ \\
\hline Outer radius of the transmitter & $R_{2}$ & $22 \mathrm{~mm}$ \\
\hline Outer radius of the receiver & $R_{4}$ & $22 \mathrm{~mm}$ \\
\hline Frequency & $f_{s}$ & $85 \mathrm{kHz}$ \\
\hline Coil distance & $\Delta d$ & $5 \sim 50 \mathrm{~mm}$ \\
\hline Misalignment & $\Delta M$ & $0 \sim 50 \mathrm{~mm}$ \\
\hline Angular alignment & $\Delta A M$ & $0 \sim 90 \mathrm{deg}$ \\
\hline Turn number of each coil & $N$ & $0 \sim 20$ \\
\hline Turn distance & $t_{d}$ & $1.15 \sim 2.15 \mathrm{~mm}$
\end{tabular}

In helical coil configuration, turns are bundled in a manner in which turn distance occupies a negligible portion of coil radius. In contrast, turns of spiral coils are separated by turn distance $t_{d}$ and thus fulfill the first and second assumptions according to (2)-(5). The study of self-inductance (L), mutual inductance $(M)$ and coupling coefficient $(K)$ proceeded by supplying the transmitting coil with a current of $1 \mathrm{~A}$ at a frequency of $f_{s}$ to assess the impact of turn number against coil distance, whereas the coil misalignment is zero. 
Table II compares the $L, M$, and $K$ simulation values for various helical coil arrangements with those determined by (2), (4), and (6). It is observed from the table that simulated values give a low relative error compared with evaluated one. Hence, the simulated values are considered as an accurate indication of the model evaluation. Likewise, Table III compares the $L, M$, and $K$ obtained from simulation with those calculated by (3), (5), and (6) for various spiral coils with a $1.45 \mathrm{~mm}$ turn distance. As mentioned in section II, the error in this approximated method (5) is significant when the distance between coils' plane is smaller than inner radius of each coil. When the turn number is 6 turns as shown in Table III, the inner radius is $13.6 \mathrm{~mm}$, which is greater than the coil distance. Hence, an error of $5.79 \%$ occurs in $M$ evaluation leading to high error of $8.97 \%$ in $K$. This error demises with increasing turn number, which reduces the inner radius of coil arrangement.

TABLE II

COMParison Between The Evaluated And Simulated Values Of Inductive Parameters For Helical Coil.

\begin{tabular}{|c|c|c|c|c|c|c|c|c|c|c|c|}
\hline \multirow{2}{*}{\multicolumn{3}{|c|}{ Coil distance }} & \multicolumn{3}{|c|}{$10 \mathrm{~mm}$} & \multicolumn{3}{|c|}{$15 \mathrm{~mm}$} & \multicolumn{3}{|c|}{$20 \mathrm{~mm}$} \\
\hline & & & Sim & Calc & Err\% & Sim & Calc & Err\% & Sim & Calc & Err\% \\
\hline \multirow{9}{*}{ 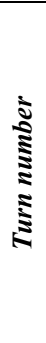 } & \multirow{3}{*}{6} & $L(\mu H)$ & 2.614 & 2.632 & 0.662 & 2.614 & 2.632 & 0.669 & 2.614 & 2.632 & 0.677 \\
\hline & & $M(\mu H)$ & 0.655 & 0.669 & 2.103 & 0.435 & 0.448 & 3.133 & 0.299 & 0.313 & 4.484 \\
\hline & & $K$ & 0.250 & 0.254 & 1.429 & 0.166 & 0.170 & 2.447 & 0.114 & 0.119 & 3.776 \\
\hline & \multirow{3}{*}{10} & $L(\mu H)$ & 5.893 & 5.962 & 1.167 & 5.893 & 5.962 & 1.174 & 5.892 & 5.962 & 1.183 \\
\hline & & $M(\mu H)$ & 1.594 & 1.625 & 1.913 & 1.048 & 1.078 & 2.882 & 0.714 & 0.743 & 4.167 \\
\hline & & $K$ & 0.271 & 0.273 & 0.739 & 0.178 & 0.181 & 1.687 & 0.121 & 0.125 & 2.947 \\
\hline & \multirow{3}{*}{14} & $L(\mu \mathrm{H})$ & 9.490 & 9.527 & 0.393 & 9.489 & 9.527 & 0.399 & 9.489 & 9.527 & 0.408 \\
\hline & & $M(\mu \mathbf{H})$ & 2.694 & 2.741 & 1.760 & 1.755 & 1.802 & 2.673 & 1.184 & 1.230 & 3.912 \\
\hline & & $\boldsymbol{K}$ & 0.284 & 0.288 & 1.360 & 0.185 & 0.189 & 2.266 & 0.125 & 0.129 & 3.487 \\
\hline
\end{tabular}

The plot of self and mutual inductances of helical coil with changing turn number is shown in Fig. 5 for various coil distances. Curves have same change manner in self-inductance as well as the mutual inductance relative to the square number of turns. In addition, self-inductance is the same as increasing the distance between the coupling coils since the system does not have a magnetic core, while mutual inductance decreases with increasing coil distance.

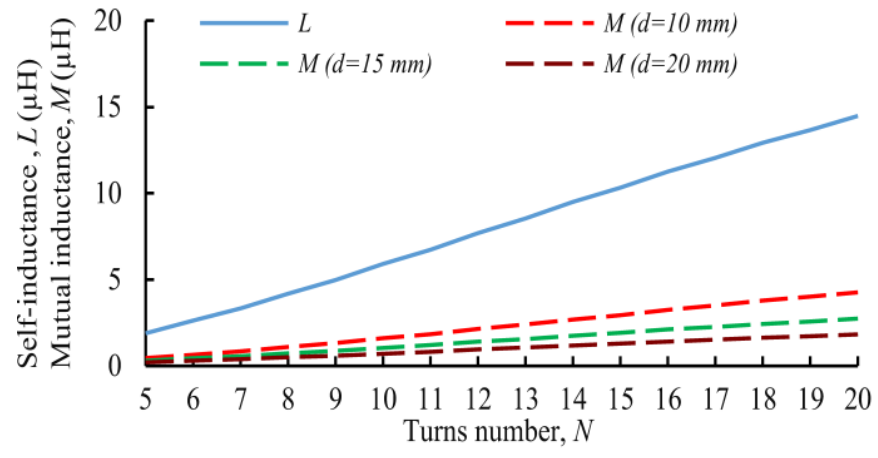

Fig. 5. Simulated values of $L$ and $M$ against $N$ for various distances of helical coil.

TABLE III

Comparison Between The Evaluated ANd Simulated Values Of Inductive Parameters For Spiral coil.

\begin{tabular}{|c|c|c|c|c|c|c|c|c|c|c|c|}
\hline \multirow{2}{*}{\multicolumn{3}{|c|}{$\begin{array}{l}\text { Coil distance } \\
t_{d}=1.45 \mathrm{~mm}\end{array}$}} & \multicolumn{3}{|c|}{$10 \mathrm{~mm}$} & \multicolumn{3}{|c|}{$15 \mathrm{~mm}$} & \multicolumn{3}{|c|}{$20 \mathrm{~mm}$} \\
\hline & & & Sim & Calc & Err\% & Sim & Calc & Err\% & Sim & Calc & Err\% \\
\hline \multirow{9}{*}{ 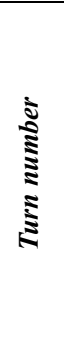 } & \multirow{3}{*}{6} & $L(\mu H)$ & 1.778 & 1.840 & 3.499 & 1.778 & 1.840 & 3.505 & 1.777 & 1.840 & 3.517 \\
\hline & & $M(\mu H)$ & 0.533 & 0.502 & 5.795 & 0.344 & 0.339 & 1.368 & 0.230 & 0.236 & 2.331 \\
\hline & & $K$ & 0.300 & 0.273 & 8.979 & 0.194 & 0.184 & 4.709 & 0.130 & 0.128 & 1.143 \\
\hline & \multirow{3}{*}{10} & $L(\mu H)$ & 2.991 & 3.064 & 2.423 & 2.991 & 3.064 & 2.430 & 2.991 & 3.064 & 2.436 \\
\hline & & $M(\mu H)$ & 0.939 & 0.915 & 2.557 & 0.595 & 0.593 & 0.398 & 0.391 & 0.400 & 2.380 \\
\hline & & $K$ & 0.314 & 0.299 & 4.866 & 0.199 & 0.194 & 2.759 & 0.131 & 0.131 & 0.051 \\
\hline & \multirow{3}{*}{14} & $L(\mu H)$ & 3.447 & 3.502 & 1.575 & 3.447 & 3.502 & 1.578 & 3.447 & 3.502 & 1.587 \\
\hline & & $M(\mu H)$ & 1.069 & 1.050 & 1.748 & 0.672 & 0.671 & 0.232 & 0.438 & 0.449 & 2.333 \\
\hline & & $K$ & 0.310 & 0.300 & 3.270 & 0.195 & 0.192 & 1.781 & 0.127 & 0.128 & 0.737 \\
\hline
\end{tabular}

Fig. 6 plots self-inductance, as well as mutual inductance with the change of turn number that is limited to 15 , turns with $t_{d}$ of $1.45 \mathrm{~mm}$ due to the constraint of spiral coil geometry. The plot shows that both self and mutual inductances curves having the same response and their values are smaller than in the helical coil. This is because the overall flux linkage is reduced because the area of the internal turns is lower than that of the external one. The analysis of both self and mutual inductances against turn distance for spiral coil is shown in Fig. 7 when $N=10$ and $d=10 \mathrm{~mm}$. 


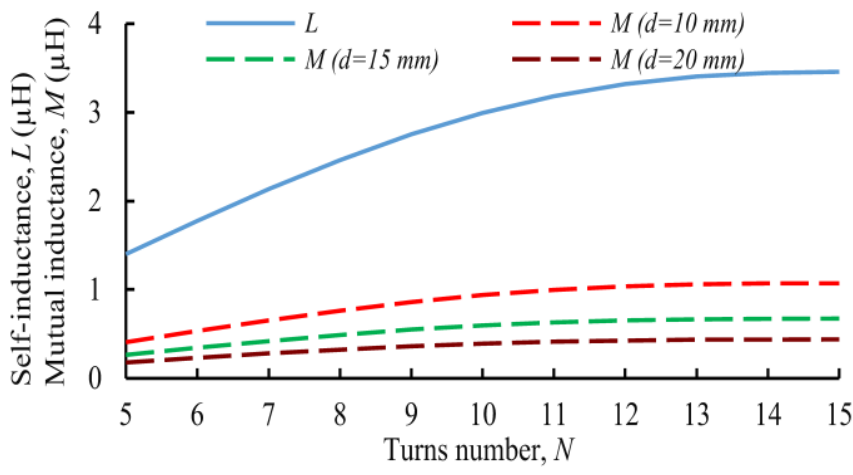

Fig. 6. Simulated values of $L$ and $M$ against $N$ for various distances of spiral coil with $t_{d}=1.45 \mathrm{~mm}$.

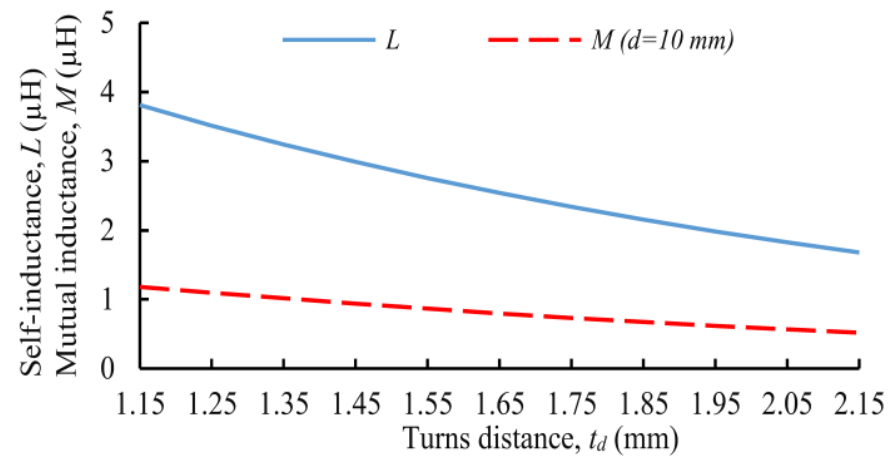

Fig. 7. Simulated values of $L$ and $M$ against $t_{d}$ of the spiral arrangement with $N$ $=10$ and $d=10 \mathrm{~mm}$.

The analysis of three different misalignments for helical and spiral coil couplings is described in Figs. 8-10, with the arrangement of $N=10$ for both coils and $t_{d}=1.45 \mathrm{~mm}$ for spiral one. This arrangement tends to be a helical coil with reducing turn distance, which explains higher $L$ and $M$ as the turn distance of the spiral coil decreases.

For vertical misalignment, Fig. 8 displays the coupling coefficient of both coil arrangement with the change of coil distance. The $K$ value of spiral arrangement is $12 \%$ to $19 \%$ greater than the helical one, while the coil distance rises from 5 to $15 \mathrm{~mm}$, which means that WPTS has a higher efficiency when using spiral coils.

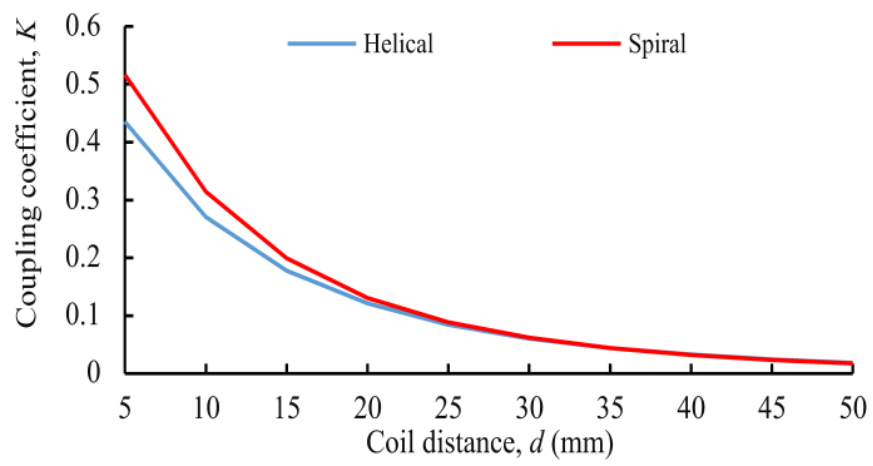

Fig. 8. Simulated values of $K$ against coil distance for helical and spiral coils.

Fig. 9 illustrates the coupling coefficient against horizontal misalignment of the two coil-coupling types on planes $10 \mathrm{~mm}$ apart. In the first portion, the coupling coefficient profile decreases with increasing misalignment, then at a specific misalignment value it becomes zero due to flux cancelation. Beyond the null point, the coupling coefficient is negative because the amount of flux entering the secondary coil from the bottom is zero or less than that entering from the top [22]. The $K$ value of spiral arrangement is $5 \%$ to $16 \%$ greater than the helical one, whereas the misalignment increases from zero to $15 \mathrm{~mm}$. In addition, the decrease in $K$ for spiral arrangement when $d=15 \mathrm{~mm}$ is $61 \%$ which is not much different from $59 \%$ of the helical one. Instead, the sensitivity of $K$ to the misalignment at $15 \mathrm{~mm}$ causes a decrease of $K$ by $40 \%$ and $46 \%$ for helical and spiral coils, respectively.

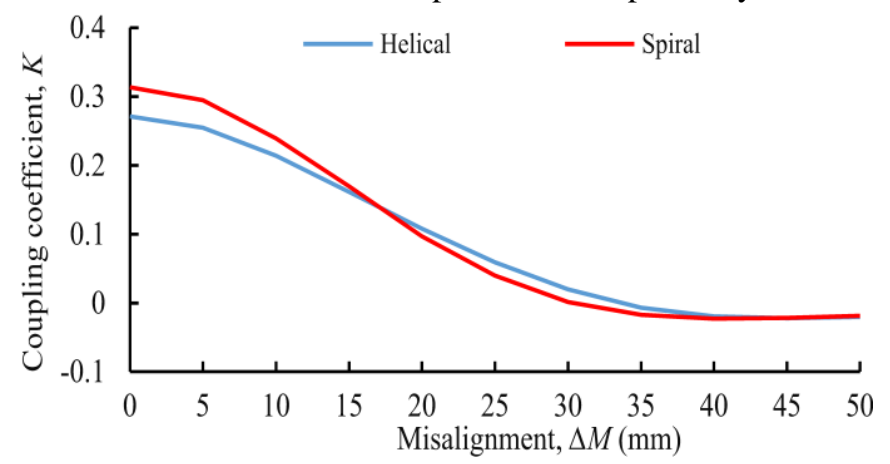

Fig. 9. Simulated values of $K$ against horizontal misalignment for helical and spiral coils.

Fig. 10 indicates the difference in coupling coefficient to the angular misalignment of both coil-coupling placed on 25 $\mathrm{mm}$ separate planes where the receiving coil rotates around its center. The coupling coefficient of both arrangements increases until 40 degrees because of increasing the amount of linking flux entering the per unit area of the secondary coil. The spiral coil has a higher coupling coefficient value than the helical one. Moreover, the increase of $K$ is $5.8 \%$ and $2.2 \%$ for helical and spiral coils, respectively.

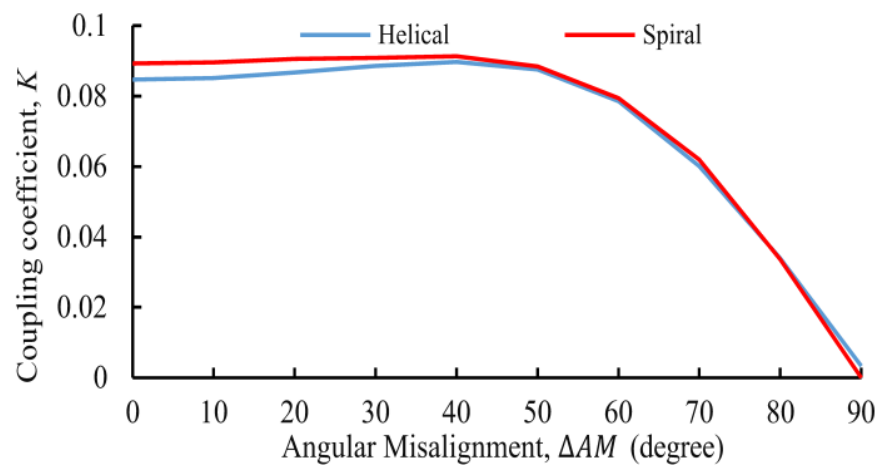

Fig. 10. Coupling coefficient against angular misalignment for helical and spiral coils.

\section{INDUCTIVE ChaRACTERISTICS OF CORED COILS}

Magnetic cores are used to increase coupling characteristics between charging coils. Also, it has been used 
as an alternative way to reduce the self-inductance of the coil arrangement. Four cores (square I-core, circular I-core, Ucore, and E-core) with high magnetic permeability of $\mu_{r}=$ 2000 are used to analyze the inductive parameter as shown in Fig. 2. The dimension and geometry of the cores for two coil forms are seen in Fig. 11.

As shown in Fig. 2, the square and circular I-cores are represented by a black plate over which the coils are placed where the dimensions $\mathrm{C}$ and $\mathrm{B}$ are set to zero. The square length and diameter of the circle are $50 \mathrm{~mm}$ as shown from the side view in Fig. 11. The U-core is represented by a green rim around the base plate when dimension $\mathrm{C}$ is set to zero. To perform E-core, a green section with a width of $\mathrm{C}$ that depends on the number and distance of turns is added to the U-core. Further to the previous Section, the helical and spiral coils, with $N=10, d=10 \mathrm{~mm}$ and $t_{d}=1.45 \mathrm{~mm}$ for spiral coil, are considered in evaluating the inductive parameters of the cored arrangement. The magnetic parameters of four simulated cores are performed by changing coil distance and horizontal misalignment.

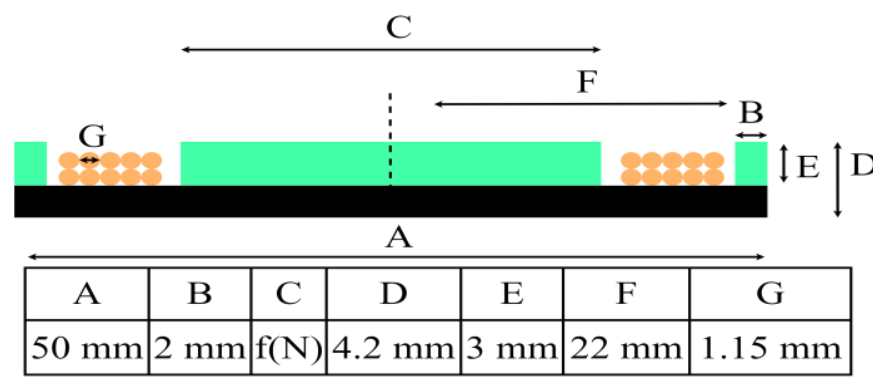

(a)

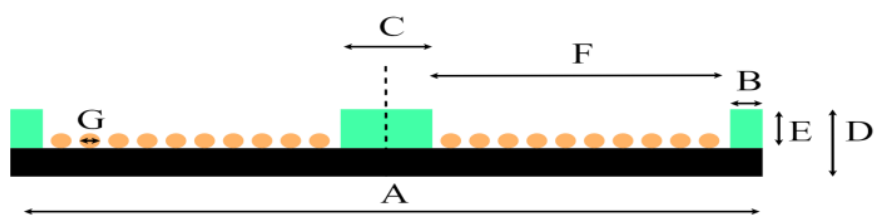

\begin{tabular}{|c|c|c|c|c|c|c|}
\hline A & B & C & D & E & F & G \\
\hline $50 \mathrm{~mm}$ & $2 \mathrm{~mm}$ & $\mathrm{f}\left(\mathrm{N}, \mathrm{t}_{\mathrm{d}}\right)$ & $4.2 \mathrm{~mm}$ & $3 \mathrm{~mm}$ & $22 \mathrm{~mm}$ & $1.15 \mathrm{~mm}$ \\
\hline
\end{tabular}

(b)

Fig. 11. The layout of the ferrite core for (a) the helical coil and (b) the spiral coil.

\section{A. Variation in Coil Distance for Both Coil Winding}

Fig. 12 illustrates the change of self and mutual inductances in relation to the helical coil distance shown in Fig. 11 (a), including various core structures. Compared to other core arrangements, E-core presents higher $L$ and $M$ values and achieves approximately $136 \%$ and $210 \%$ higher self and mutual inductances than non-core structures, respectively. In Fig. 13, the coupling coefficient against coil distance again shows that the E-core is the best in coupling profile and achieves about $31 \%$ greater than coreless arrangements.

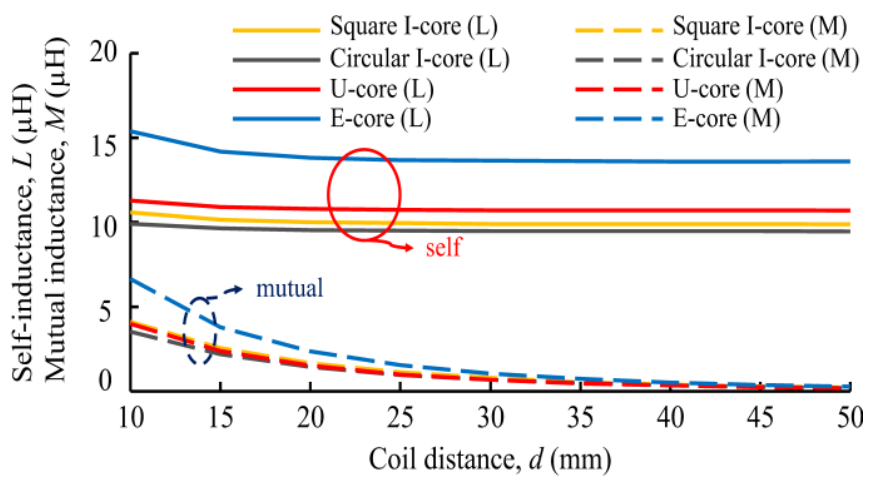

Fig. 12. Simulated values of $L$ and $M$ against coil distances of the helical coil with various cores setup.

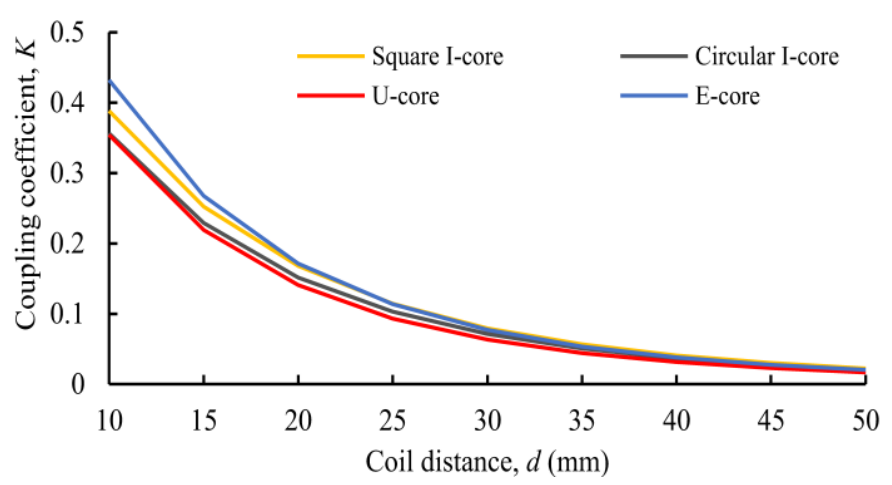

Fig. 13. Simulated values of $K$ against coil distances of the helical coil with various cores setup.

Figs. 12 and 14 show that self-inductance is independent on the coil distance in contrast to mutual inductance, which is inversely proportional to coil distance. Moreover, Figs. 14 and Fig. 15 show the magnetic characteristics against spiral coil distance with different core configurations that have the same pattern of helical coil coupling. Similarly, E-core has higher $L$, $M$, and $K$ than coreless structures by $126 \%, 220 \%$, and $41 \%$, respectively. Additionally, the E-core coupling coefficient of spiral arrangement is better than the helical one, which achieves an increase of $K$ by $34 \%$.

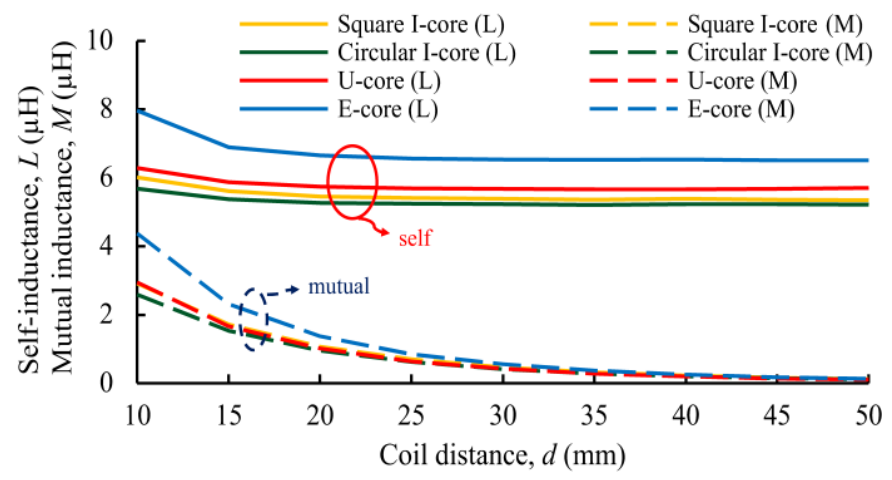

Fig. 14. Simulated values of $L$ and $M$ against coil distances of the spiral coil with various cores setup. 


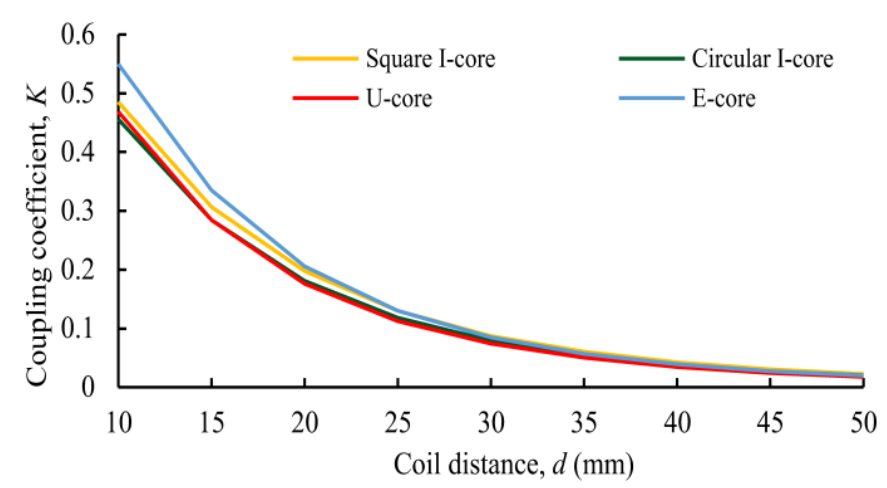

Fig. 15. Simulated values of $K$ against coil distances of the spiral coil with various cores setup.

\section{B. Variation in Horizontal Misalignment for Both Coil Winding}

Fig. 16 plots the coupling coefficient against the misalignment of helical coil including different core configurations. At a small misalignment, the E-core shows higher $K$ values, while at a larger one the square I-core values are higher. Core responses to the misalignment are the same as those indicated in non-core setups. Instead, the coupling coefficient of the E-core is $43 \%$ higher than the coreless arrangement, whereas the misalignment ranges from zero to $15 \mathrm{~mm}$.

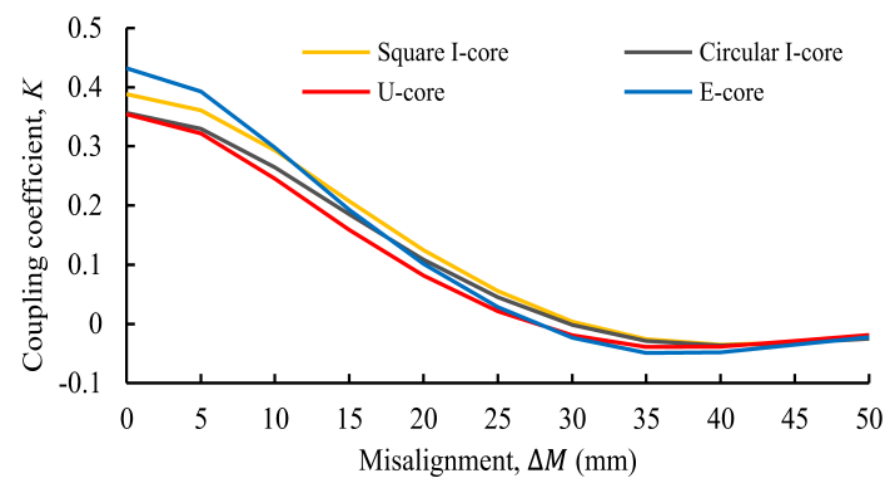

Fig. 16. Simulated values of $K$ against misalignment of the helical coil with various cores setup.

Likewise, Fig. 17 presents the coupling coefficient for different cores of spiral coil with horizontal misalignment. It is shown in Figs. 16 and 17 that the coupling coefficient of each core structure of the spiral coil is greater than the helical one and has the same changing pattern as illustrated in Fig. 9. Moreover, the E-core coupling coefficient of the spiral configuration is $54 \%$ higher than the non-core arrangement and $26 \%$ higher than the helical cored one, while the misalignment varies from zero to $15 \mathrm{~mm}$. the analysis proves that the ferrite cores have the best results in coupling coefficient, particularly in the case of spiral coil setups.

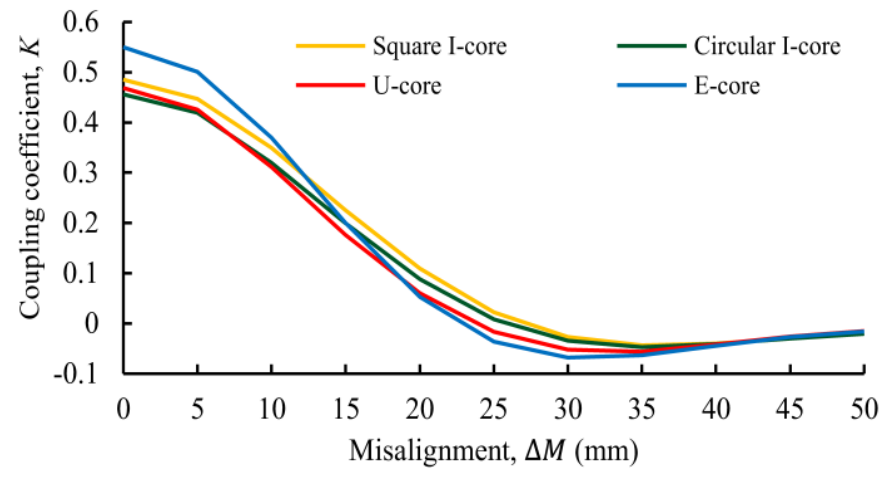

Fig. 17. Simulated values of $K$ against misalignment of the spiral coil with various cores setup.

\section{CONCLUSION}

Coil arrangements have an important role in WPTS since different core and winding shapes have been developed to charge EVs. This paper presents theoretical analyses of the inductive characteristics for coreless helical and spiral coils that have been supported by COMSOL software. It has been found that the formulas provide an excellent approximation according to the change of coil distance and turn number for various coil arrangements. The paper also discussed variations in geometric parameters and misalignment of the coils, including magnetic characteristics. The results show that, although the spiral coil arrangement has reduced self and mutual inductances, it also has higher coupling features than the helical one for all misalignment cases. Moreover, the higher coupling coefficient is found at 40 degrees for both coil arrangements in the case of angular frequency. Four shapes of the ferrite core were used in both helical and spiral coils to improve magnetic parameters compared to the coreless counterpart. Furthermore, it has been found that the E-core presents a higher sensitivity to the coupling coefficient with a change in coil distance and misalignment, especially in the case of spiral type.

\section{Authors CONTRIBution}

Sahar Sidky Kaddah and Mohammad E. M. Rizk conceived of the presented idea. Ahmed M. Elbeshbeshy developed the theory and performed the computations. Mohammad E. M. Rizk and Ahmed M. Elbeshbeshy verified the analytical methods. Sahar Sidky Kaddah and Mohammad E. M. Rizk have suggested Ahmed M. Elbeshbeshy to investigate the comparison between calculated and simulated inductive characteristics and supervised the findings of this work. All authors discussed the results and contributed to the final manuscript. Ahmed M. Elbeshbeshy wrote the manuscript with support from Sahar Sidky Kaddah and Mohammad E. M. Rizk.

\section{REFERENCES}

[1] S. Niu, H. Xu, Z. Sun, Z. Y. Shao, and L. Jian, "The state-of-the-arts of wireless electric vehicle charging via magnetic resonance: principles, standards and core technologies," Renewable and Sustainable Energy Reviews, vol. 114. Elsevier Ltd, p. 109302, 01-Oct-2019.

[2] A. Ahmad, M. S. Alam, and R. Chabaan, "A Comprehensive Review of Wireless Charging Technologies for Electric Vehicles," IEEE Trans. 
Transp. Electrif., vol. 4, no. 1, pp. 38-63, Nov. 2017.

[3] M. İnci, M. Büyük, M. H. Demir, and G. İlbey, "A review and research on fuel cell electric vehicles: Topologies, power electronic converters, energy management methods, technical challenges, marketing and future aspects," Renew. Sustain. Energy Rev., vol. 137, p. 110648, Mar. 2021.

[4] C. Liu, C. Jiang, and C. Qiu, "Overview of coil designs for wireless charging of electric vehicle," in 2017 IEEE PELS Workshop on Emerging Technologies: Wireless Power Transfer, WoW 2017, 2017.

[5] Z. Yi, M. Li, B. Muneer, G. He, and X.-X. Yang, "Self-Resonant Antisymmetric Planar Coil for Compact Inductive Power Transfer System Avoiding Compensation Circuits," IEEE Trans. Power Electron., pp. 1-1, Oct. 2020.

[6] S. Y. R. Hui, W. Zhong, and C. K. Lee, "A critical review of recent progress in mid-range wireless power transfer," IEEE Transactions on Power Electronics, vol. 29, no. 9. Institute of Electrical and Electronics Engineers Inc., pp. 4500-4511, 2014.

[7] A. Ramezani and M. Narimani, "Optimized Electric Vehicle Wireless Chargers with Reduced Output Voltage Sensitivity to Misalignment," IEEE J. Emerg. Sel. Top. Power Electron., vol. 8, no. 4, pp. 3569-3581, Dec. 2020.

[8] C. Panchal, S. Stegen, and J. Lu, "Review of static and dynamic wireless electric vehicle charging system," Engineering Science and Technology, an International Journal, vol. 21, no. 5. Elsevier B.V., pp. 922-937, 01Oct-2018.

[9] A. K. RamRakhyani, S. Mirabbasi, and M. Chiao, "Design and optimization of resonance-based efficient wireless power delivery systems for biomedical implants," IEEE Trans. Biomed. Circuits Syst., vol. 5, no. 1, pp. 48-63, Feb. 2011.

[10] C. Qiu, K. T. Chau, C. Liu, W. Li, and F. Lin, "Quantitative comparison of dynamic flux distribution of magnetic couplers for roadway electric vehicle wireless charging system," in Journal of Applied Physics, 2014, vol. 115, no. 17, p. 17A334.

[11] J. C. Maxwell, "Treatise on Electricity and Magnetism, 2 vols., reprint by Dover," New York, 1954.

[12] C. Akyel, S. I. Babic, and M. M. Mahmoudi, "Mutual inductance calculation for noncoaxial circular air coils with parallel axes," Prog. Electromagn. Res., vol. 91, pp. 287-301, 2009.

[13] H. M. Greenhouse, "Design of Planar Rectangular Microelectronic Inductors," IEEE Trans. Parts, Hybrids, Packag., vol. 10, no. 2, pp. $101-109,1974$

[14] H. A. Wheeler, "Simple inductance formulas for radio coils," Proc. Inst. Radio Eng., vol. 16, no. 10, pp. 1398-1400, 1928.

[15] S. S. Mohan, M. D. M. Hershenson, S. P. Boyd, and T. H. Lee, "Simple accurate expressions for planar spiral inductances," IEEE J. Solid-State Circuits, vol. 34, no. 10, pp. 1419-1420, 1999.

[16] S. Babic, F. Sirois, C. Akyel, G. Lemarquand, V. Lemarquand, and R. Ravaud, "New formulas for mutual inductance and axial magnetic force between a thin wall solenoid and a thick circular coil of rectangular cross-section," IEEE Trans. Magn., vol. 47, no. 8, pp. 2034-2044, 2011.

[17] S. Babic, F. Sirois, C. Akyel, and C. Girardi, "Mutual inductance calculation between circular filaments arbitrarily positioned in space: Alternative to grover's formula," IEEE Trans. Magn., vol. 46, no. 9, pp. 3591-3600, Sep. 2010
[18] J. T. Conway, "Inductance calculations for noncoaxial coils using bessel functions," IEEE Trans. Magn., vol. 43, no. 3, pp. 1023-1034, Mar. 2007.

[19] F. W. Grover, Inductance calculations: working formulas and tables. Courier Corporation, 2004.

[20] "Comsol Multiphysics, 'Documentation Set', version 5.4, www. comsol.com.".

[21] G. A. Covic and J. T. Boys, "Modern trends in inductive power transfer for transportation applications," IEEE J. Emerg. Sel. Top. Power Electron., vol. 1, no. 1, pp. 28-41, 2013.

[22] K. Aditya, V. K. Sood, and S. S. Williamson, "Magnetic characterization of unsymmetrical coil pairs using archimedean spirals for wider misalignment tolerance in ipt systems," IEEE Trans. Transp. Electrif., vol. 3, no. 2, pp. 454-463, 2017.

[23] K. N. Mude, M. Bertoluzzo, and G. Buja, "Inductive characteristics of different coupling setups for wireless charging of an electric city-car," in 2014 IEEE International Electric Vehicle Conference, IEVC 2014, 2014.

[24] S. Li and C. C. Mi, "Wireless power transfer for electric vehicle applications," IEEE J. Emerg. Sel. Top. Power Electron., vol. 3, no. 1, pp. 4-17, Mar. 2015.

[25] K. Fotopoulou and B. W. Flynn, "Wireless power transfer in loosely coupled links: Coil misalignment model," IEEE Trans. Magn., vol. 47, no. 2 PART 2, pp. 416-430, Feb. 2011.

[26] K. B. Kim, E. Levi, Z. Zabar, and L. Birenbaum, "Mutual inductance of noncoaxial circular coils with constant current density," IEEE Trans. Magn., vol. 33, no. 5 PART 3, pp. 4303-4309, 1997.

[27] S. Raju, R. Wu, M. Chan, and C. P. Yue, "Modeling of mutual coupling between planar inductors in wireless power applications," IEEE Trans. Power Electron., vol. 29, no. 1, pp. 481-490, 2014.

\section{Title Arabic:}

\section{تحليل الخصائص الحثية لأنظمة متنوعة من الملفات الحلزونية

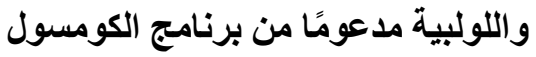

\section{Arabic Abstract:}

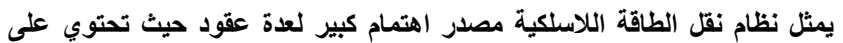

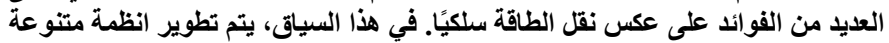

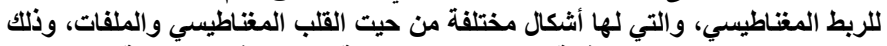

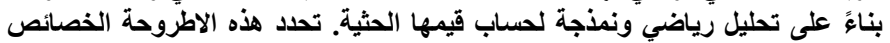

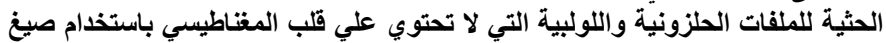

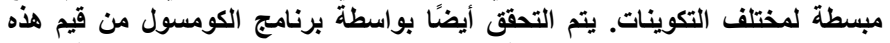

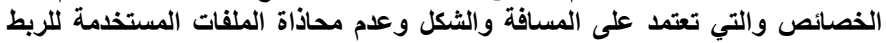

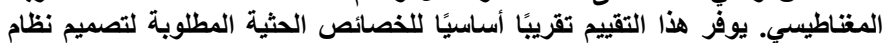

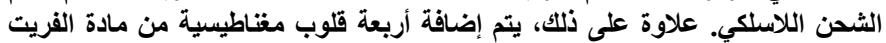

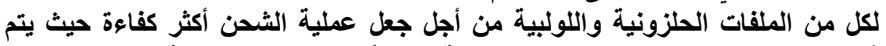

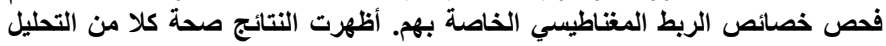
النظري والمحاكاة. 